from the Neandertal type specimen. Proc. Natl Acad. Sci. USA 96, 5581-5585 (1999).

25. Krings, M. et al. A view of Neandertal genetic diversity. Nature Genet. 26, 144-146 (2000).

26. Nordborg, M. On the probability of Neanderthal ancestry. Am. J. Hum. Genet. 63, 1237-1240 (1998).

27. Pääbo, S. Human evolution. Trends Cell Biol. 9, M13-M16 (1999).

28. Stringer, C. Modern human origins: progress and prospects. Phil. Trans. R. Soc. Lond. B 357, 563-579 (2002).

29. Deinard, A. \& Kidd, K. Evolution of a HOXB6 intergenic region within the great apes and humans. J. Hum. Evol. 36, 687-703 (1999).

30. Kaessmann, H., Wiebe, V., Weiss, G. \& Pääbo, S. Great ape DNA sequences reveal a reduced diversity and an expansion in humans. Nature Genet. 27, 155-156 (2001).

31. Lewontin, R. C. The problem of genetic diversity. Evol. Biol. 6, 381-398 (1972).

32. Kaessmann, H., Heissig, F., von Haesler, A. \& Pääbo, S. DNA sequence variation in a non-coding region of low

recombination on the human X chromosome. Nature Genet. 22, 78-81 (1999).

33. Harris, E. E. \& Hey, J. X chromosome evidence for ancient human histories. Proc. Natl Acad. Sci. USA 96, 3320-3324 (1999).

34. Yua, N. \& Li, W.-H. No fixed nucleotide difference between Africans and non-Africans at the pyruvate dehydrogenase E1 $\alpha$-subunit locus. Genetics 155, 1481-1483 (2000).
35. Cavalli-Sforza, L. L., Menozzi, P. \& Piazza, A. The History and Geography of Human Genes (Princeton Univ. Press, Princeton, NJ, 1993).

36. Risch, N., Burchard, E., Ziv, E. \& Tang, H. Categorization of humans in biological research: genes, race and disease. Genome Biol. 3, 2007.1-2007.12 (2002).

37. Tomasello, M. \& Call, J. Primate Cognition (Oxford Univ. Press, New York, 1997)

38. Whiten, A. et al. Cultures in chimpanzees. Nature 399, 682-685 (1999).

39. Eichler, E. E. Recent duplication, domain accretion and the dynamic mutation of the human genome. Trends Genet. 17, 661-669 (2001).

40. Enard, W. et al. Intra- and interspecific variation in primate gene expression patterns. Science 296, 340-343 (2002).

41. Enard, W. et al. Molecular evolution of FOXP2, a gene involved in speech and language. Nature 418, 869-872 (2002).

42. Jackson, A. P. et al. Identification of microcephalin, a protein implicated in determining the size of the human brain. Am. J. Hum. Genet. 71, 136-142 (2002).

\section{Acknowledgements}

My work is funded by the Max Planck Society, the Bundesministerium für Bildung und Forschung and the Deutsche Forschungsgemeinschaft. I thank B. Cohen, H. Kaessmann, D. Serre, M. Stoneking, C. Stringer, L.

Vigilant and especially D. Altshuler for helpful comments on the manuscript.

\title{
Nature, nurture and human disease
}

\section{Aravinda Chakravarti ${ }^{\star}$ \& Peter Little $\dagger$}

${ }^{*}$ McKusick-Nathans Institute of Genetic Medicine, Johns Hopkins University School of Medicine, 600 North Wolfe Street, Jefferson Street Building, 2-109, Baltimore, Maryland 21287, USA (e-mail:aravinda@jhmi.edu)

$\dagger$ School of Biotechnology and Biomolecular Sciences, University of New South Wales, Sydney, New South Wales 2052, Australia (e-mail:p.little@unsw.edu.au)

What has been learnt about individual human biology and common diseases 50 years on from the discovery of the structure of DNA? Unfortunately the double helix has not, so far, revealed as much as one would have hoped. The primary reason is an inability to determine how nurture fits into the DNA paradigm. We argue here that the environment exerts its influence at the DNA level and so will need to be understood before the underlying causal factors of common human diseases can be fully recognized.

"We used to think our fate was in our stars. Now we know, in large measure, our fate is in our genes.” J. D. Watson, quoted in Time magazine, 20 March 1989 (ref. 1).

$\mathrm{T}$ he double helix, in its simplicity and beauty, is the ultimate modern icon of contemporary biology and society. Its discovery provided the bridge between the classical breeding definition and the modern functional definition of genetics, and permanently united genetics with biochemistry, cell biology and physiology. The DNA structure provided an immediate explanation for mutation and variation, change, species diversity, evolution and inheritance. It did not, however, automatically provide a mechanism for understanding how the environment interacts at the genetic level.

\section{One gene, one disease}

Recognition that genes have a role in human disease dates back to the rediscovery of the rules that govern the inheritance of genes by Gregor Mendel — the so-called Mendelian

laws of inheritance. So far, human geneticists have been most successful at understanding single-gene disorders, as their biological basis, and thus presumed action, could be predicted from inheritance patterns. Mendelian diseases are typically caused by mutation of a single gene that results in an identifiable disease state, the inheritance of which can readily be traced through generations.

The landmark sequencing of the human genome provided some important lessons about the role of genes in human disease. Notably, mutations in specific genes lead to specific biological changes, and rarely do mutations in multiple genes lead to an identical set of characteristics that obey 'Mendelian inheritance'. Additionally, sequence diversity of mutations is large and, consequently, individual mutations are almost always rare, showing relatively uniform global distributions.
But a few exceptions do exist. Some recessive mutations (mutations that influence a person only if both copies of the gene are altered) are surprisingly common in specific populations. This defiance of general mutation patterns arises either from chance increases in frequency in isolated populations, such as in the Old Order Amish ${ }^{2}$, or from the protective effect of a deleterious mutation in a single copy, such as the genetic mutation that on the one hand causes sicklecell anaemia, but on the other hand offers protection against malaria ${ }^{3}$. These examples show that human history, geography and ecology of a particular people are relevant to understanding their present-day molecular disease burden ${ }^{4}$.

For over 90 years, the association between DNA mutations and a vast variety of single-gene disorders has repeatedly emphasized the notion that human disease results from faults in the DNA double helix (see, for example, the Online Mendelian Inheritance in Man database at www.-ncbi.nlm.nih.gov/omim/, which provides a catalogue of human genes and genetic disorders). Is it then too extrapolative to suggest that all diseases and traits, each of which has some familial and imputed inherited component, will be caused by a corrupted piece of double helix?

\section{Is our fate encoded in our DNA?}

Is Watson's genetic aphorism of human disease really true? The excitement of genetics, and the perceived medical importance of the human genome sequence, is pegged to the promise of an understanding of common chronic disease and not rare Mendelian diseases. In theory, one might hope that approaches used successfully to identify single-gene diseases could simply be applied to the common causes of world-wide morbidity and mortality, such as cancer, heart disease, psychiatric illness and the like. This would enable a boon for diagnosis, understanding and the eventual treatment of these common maladies ${ }^{5}$.

The reality is that progress towards identifying common disease mutations has been slow, and only recently have there been some successes ${ }^{6}$. It is now appreciated that although genes are one contributor to the origin of common diseases, the mutations they contain must have properties that are different from the more familiar, deterministic features of single-gene mutations. Indeed, the underlying genes are likely to be numerous, with no single gene having a major role, and mutations within these genes being common and imparting small genetic effects (none of which are either necessary or sufficient ${ }^{7}$ ).

Moreover, there is a suspicion that these mutations both interact with one another and with the environment and lifestyle, although the molecular specificity of inter- 
actions is unproven ${ }^{8}$. To complicate matters, common disorders frequently show large population differences that have led to health disparities and, as is becoming more evident, the incidence of these disorders can show significant changes over time? .

\section{Interplay of DNA and environment}

The inability of geneticists to easily identify common disease genes has been seen as a vindication of the importance of nurture. This is too simplistic; the influence of nature and nurture cannot be neatly divided, as it is clear that nurture is important to biology through its actions on DNA and its products. The environment must affect the regulation of critical genes by some mechanism and so, seen another way, mutations are not the only agent for altering gene function.

The scientific literature of cancer research reveals that despite having heterogeneous origins - both inherited and acquired - a specific tumour develops only from altering the expression (activity) of specific sets of genes ${ }^{10}$. That is, a variety of exposures and mutations collaborate to change the activity of specific genes and, consequently, interrupt precise aspects of cell metabolism. The regulation of circadian rhythm is another example of how external environmental cues influence DNA functions ${ }^{11}$.

Thus, the double helix inevitably interacts with the environment, directly and indirectly, to predispose or protect us from disease. If perturbations of multiple genes contribute to a disorder, then the activities of these genes can be affected by any combination of mutation and environmental exposure altering their function. It is our opinion that genes have a stronger, maybe even a pervasive, role in all diseases and traits, with the understanding that it is the collective action of genes and nurture that underpins ultimate disease outcome.

Rather than dismissing the role of environment, our view embraces it directly, and, by that, expands the meaning of the term 'genetic'. It also emphasizes the work that remains to be done to understand gene regulation and, in particular, how genes and their products are modulated by external cues and how homeostasis is disrupted in human disease. Human beings are each the product of a unique genome and a unique set of experiences. Both need to be understood to intervene effectively in disease causation.

\section{Implications for medicine}

What does this mean in practice? The assessment of the quantitative role of genes in human traits is derived largely from studies on identical and fraternal twins (Fig. 1). By this measure, all common disorders have a 'genetic' basis, but the contribution varies from slight in some cancers and multiple sclerosis,

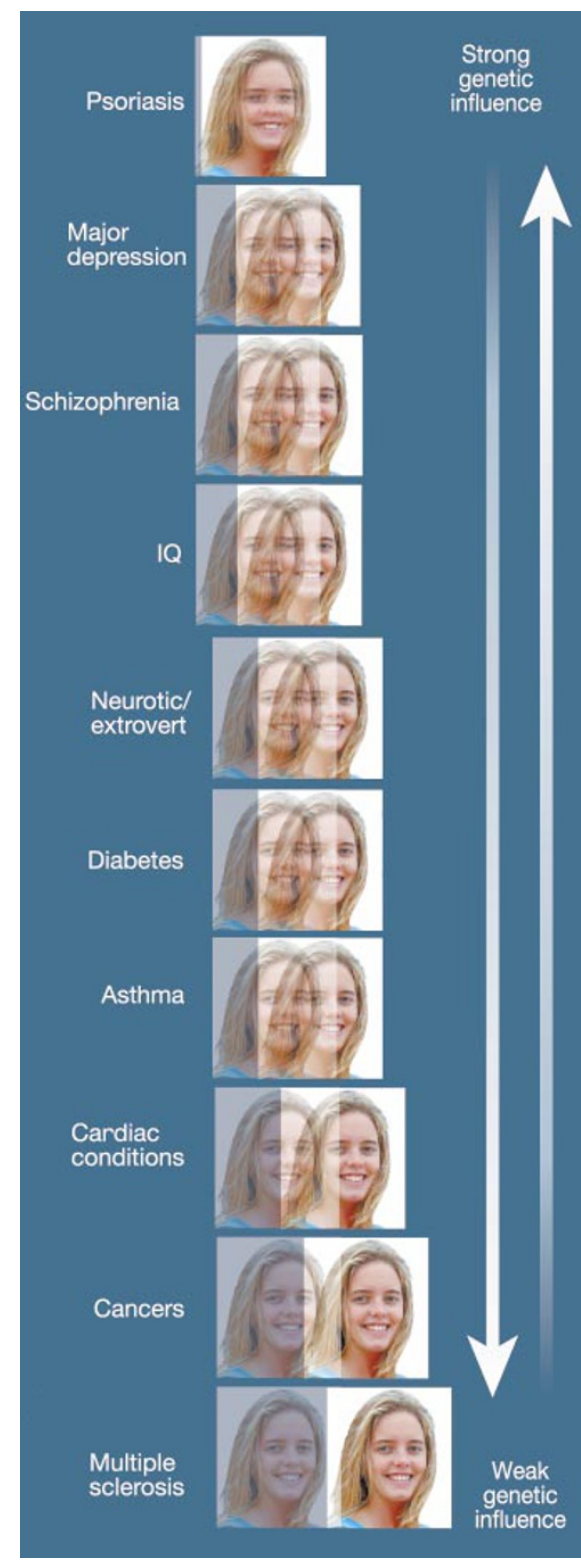

Figure 1 Studies of identical twins have revealed that some conditions, such as psoriasis, have a strong genetic component and are less influenced by environmental and lifestyle factors - identical twins are more likely to share these diseases. But other conditions, such as multiple sclerosis, are only weakly influenced by genetic makeup and therefore twins may show differences depending on their exposure to various environmental factors.

to moderate in diabetes, heart diseases, migraine and asthma, to high in disorders such as psoriasis ${ }^{12}$. Critically, the discordance between identical twins - where twins show different diseases despite being genetically identical — illustrates the influence of exogenous factors, but does not prove the lack of influence of genes: of course, environmental factors over a lifetime affect an individual's chance of developing disease.

Let us assume, for the sake of argument, that all of the relevant genetic and environmental factors are identified that lead to a disease. Appreciating the relationship of genetic variation and environment suggests that a number of presently fashionable ideas about genetics are simplistic; two in particular are the 'bar code' view of genetic diagnosis and the 'right medicine for the right patients'.

Common genetic variations are essentially binary - either an adenine or guanine base, or a cytosine or thymine base - at a given position in the sequence. Unfortunately, this leads to a tendency to define genetic individuality as a binary pattern, a so-called 'bar code' for each individual. Some genetic variants convey susceptibility to a disease, but they typically convey risk rather than certainty of being afflicted with a condition.

Knowledge based on the sequence could have significant public health implications, and even be predictive at the population level. But a human DNA bar code would provide uncomfortable, perhaps even intolerable, knowledge of likely outcomes, with no certainty, only probabilities. Most individuals, we suspect, are ill equipped to deal with the knowledge that they have a 50 per cent chance of succumbing to an illness; equally, society has had great difficulty in knowing how to respond to such information, hence the concerns regarding genetic discrimination ${ }^{13}$. The reality is that the genetic bar code is weakly predictive and individuals may find this threatening, life enhancing or just irrelevant; in any event, much work is needed to enable the predictive revolution in medicine.

Human genetic individuality has forced the recognition that medicine has to refocus on the individual. This has been the rallying cry, particularly within the pharmaceutical business, of pharmacogenomics (the application of genome-scale understanding to the development of medicines), and there is no doubt that understanding of the variation within drug-metabolizing enzymes has exploded in the past 20 years ${ }^{14}$. The underpinning idea is enormously attractive — if genetic analysis of key DNA variations can be used to understand how individuals might respond to drugs, then it could be possible to eliminate the difficult, sometimes lethal, hit-and-miss approaches to medication that are a necessary feature of present medical practice.

Unfortunately, the influence of lifestyle is just as much a feature of drug response as it is of any other genetically influenced condition. The classic case of the influence of drinking grapefruit juice on the levels of many drugs ${ }^{15}$ illustrated that there can be no such thing as 'the patient', because the patient is living in a complex world that changes by the minute. Once again, predictions for the population do not have the same predictive power for individuals.

\section{Future challenges}

The challenges that lifestyle presents to genetic studies are considerable. We believe 
that the next 50 years will bring a genuine revolution of far greater individual significance than that delivered by genetics over the past 50 years. This is because lifestyle can conceivably be analysed, and in so doing, it should be possible to develop a genuinely personalized medicine.

Researchers can now think seriously about how to identify lifestyle influences: such studies will have to be on an unprecedented scale and one of the first of these, proposed to comprise 500,000 individuals in the United Kingdom, has already started ${ }^{16}$. These kinds of studies are a bold venture into relatively uncharted territory and face substantial technical, biological and scienceculture challenges.

Scientifically, it is necessary to understand a deceptively simple equation: genes + environment $=$ outcome. The difficulty here is the uncertainty surrounding both terms in the equation; ideally, one set of genetic factors will interact with one set of environmental influences to produce identical outcomes, but it is unknown whether this is always going to be the case. A far more difficult relationship would exist if multiple genetic factors interacted with multiple environments to achieve the same outcome. The example of glutathione $S$-transferase mutations, smoking and incidence of lung cancer ${ }^{17}$ shows it is possible to detect some interactions, but it is unclear how, or even if, statistical methods might be developed for addressing the more complex possibilities.

Perhaps the greatest unknown in undertaking these projects is human psychology; the consequences of smoking have been known for many decades, but people still smoke. Advice does not imply acceptance. How to turn knowledge into practical outcomes must be an increasing focus of attention for both researchers and funding agencies.

Psychology is also in play in the initial decision to undertake this research; for researchers, funding agencies and politicians there is great risk implicit in undertaking a hugely expensive project with complex outcome. People would like to live in a simpler world, with simpler decisions, but the vision of such a project is enormous: once complete, as much will be known about the origins of human disorders as can be discovered by using such epidemiological and genetic studies. Perhaps more important, the beginnings of a new medicine will emerge, one focused uniquely and completely upon the individual, upon the combination of genetic uniqueness and personal choices that are the very essence of individual lives.

If we are collectively bold in our present decisions and accept the risk of action, a world can be created where medicine is a guide, not a place of last resort. If the past 50 years has seen the revolution of DNA, then the revolution cannot be completed without an appreciation of both genetic and environmental individuality; only then will individuals understand the meaning of their inheritance.

doi:10.1038/nature01401

1. Jaroff, L. The gene hunt. Time 20 March, 62-67 (1989)

2. Arcos-Burgos, M. \& Muenke, M. Genetics of population isolates. Clin. Genet. 61, 233-247 (2002).

3. Aidoo, M. et al. Protective effects of the sickle cell gene against malaria morbidity and mortality. Lancet 359, 1311-1312 (2002).

4. Tishkoff, S. A. \& Williams, S. M. Genetic analysis of African populations: human evolution and complex disease. Nature Rev. Genet. 3, 611-621 (2002).

5. Chakravarti, A. Single nucleotide polymorphisms: ...to a future of genetic medicine. Nature 409, 822-823 (2001).

6. Carrasquillo, M. M. et al. Genome-wide association study and mouse model identify interaction between RET and EDNRB pathways in Hirschsprung disease. Nature Genet. 32, 237-244 (2002).

7. Cox, N. J. Challenges in identifying genetic variation affecting susceptibility to type 2 diabetes: examples from studies of the calpain-10 gene. Hum. Mol. Genet. 10, 2301-2305 (2001).
8. Sullivan, P. F. et al. Analysis of epistasis in linked regions in the Irish study of high-density schizophrenia families. Am. J. Med. Genet. 105, 266-270 (2001)

9. Boyle, J. P. et al. Projection of diabetes burden through 2050: impact of changing demography and disease prevalence in the United States. Diabetes Care 24, 1936-1940 (2001).

10. Münger, K. Disruption of oncogene/tumor suppressor networks during human carcinogenesis. Cancer Invest. 20, 71-81 (2002).

11. Panda, S., Hogenesch, J. B. \& Kay, S. A. Circadian rhythms from flies to human. Nature 417, 329-335 (2002).

12. MacGregor, A. J. et al. Twins: novel uses to study complex traits and genetic diseases. Trends Genet. 16, 131-134 (2000).

13. Wertz, D. C. Ethics watch. Nature Rev. Genet. 3, 496 (2002).

14. Evans, W. E. \& Johnson, J. A. Pharmacogenomics: the inherited basis for interindividual differences in drug response. Anпи. Rev. Genomics Hum. Genet. 2, 9-39 (2001).

15. Lown, K. S. et al. Grapefruit juice increases felodipine oral availability in humans by decreasing intestinal CYP3A protein expression. J. Clin. Invest. 99, 2545-2553 (1997).

16. Wright, A. F., Carothers, A. D. \& Campbell, H. Geneenvironment interactions- the BioBank UK study. Pharmacogenomics J. 2, 75-82 (2002).

17. Stucker, I. et al. Genetic polymorphisms of glutathione Stransferases as modulators of lung cancer susceptibility. Carcinogenesis 23, 1475-1481 (2002).

\section{The double helix in clinical practice}

\section{John I. Bell}

The Office of the Regius Professor of Medicine, University of Oxford, Oxford OX3 9DU, UK

(e-mail:regius@medsci.ox.ac.uk)

The discovery of the double helix half a century ago has so far been slow to affect medical practice, but significant transformations are likely over the next $\mathbf{5 0}$ years. Changes to the way medicine is practised and new doctors are trained will be required before potential benefits are realized.

"It is much more important to know what kind of patient has a disease than to know what kind of disease a patient has." Caleb Parry, 18th century physician, Bath.

$\mathrm{T}$ he structure of DNA established the basic framework that would develop into the field of molecular genetics. The information gleaned from this scientific endeavour continues to have a profound influence on our understanding of biological systems ${ }^{1}$. As most human diseases have a significant heritable component, it was soon recognized that the characterization of the genetic determinants of disease would provide remarkable opportunities for clinical medicine, potentially altering the way disease was understood, diagnosed and treated.

But despite the obvious potential applications to medicine, the development of significant genetic advances relevant to clinical practice could take generations. This is in marked contrast to many other medically related discoveries that occurred around the same time and which were translated rapidly into clinical practice. For instance, the development of penicillin by Ernst Chain and Howard Florey in 1941 was saving thousands of lives within months of their discovery of how to efficiently produce the antibiotic $^{2}$. Discoveries relating to disease aetiology, such as the recognition in 1950 of a relationship between smoking and lung cancer, have had a profound effect on mortality ${ }^{3}$.
This was despite the convictions of at least one distinguished statistical geneticist who argued against the causality of this observation, implying that a common genetic factor caused both lung cancer and a predilection to smoking cigarettes ${ }^{4}$ !

Although other important discoveries have had demonstrably more impact on health care at the time of their fiftieth anniversaries than has the double helix, its slower transition from discovery to clinical implementation will be balanced by its potentially profound impact across all medical disciplines. Progress has been slow, but mounting evidence suggests that, while public health and antibiotics produced important healthcare outcomes in the past 50 years, the next 50 are likely to belong to genetics and molecular medicine.

The potential impact of genetics on clinical practice has been questioned by some observers $^{5}$ who believe that the positive predictive value of genetic testing for most common disease genes will be insufficient to provide the beneficial effects seen with single-gene disorders, which affect only a tiny proportion of the population. Many advocates of genetics argue, on the other hand, that our understanding of disease is 